\title{
Multiple echocardiography abnormalities associated with endoscopic third ventriculostomy failure
}

\author{
Ashish H. Shah, MD,,2 George M. Ibrahim, MD, PhD,,2 Jun Sasaki, MD, ${ }^{3}$ John Ragheb, MD,,2 \\ Sanjiv Bhatia, MD, ${ }^{1,2}$ and Toba N. Niazi, MD ${ }^{1,2}$ \\ Divisions of ${ }^{1}$ Neurosurgery and ${ }^{2}$ Pediatric Cardiology, Nicklaus Children's Hospital; and ${ }^{3}$ Department of Neurosurgery, University \\ of Miami School of Medicine/Jackson Memorial Hospital, Miami, Florida
}

\begin{abstract}
OBJECTIVE Although endoscopic third ventriculostomy (ETV) with choroid plexus cauterization (CPC) has gained increasing prominence in the management of hydrocephalus caused by intraventricular hemorrhage of prematurity, the rates of long-term shunt independence remain low. Furthermore, limited evidence is available to identify infants who might benefit from the procedure. The authors tested the hypothesis that elevated venous pressure that results from comorbid cardiac disease might predispose patients to ETVICPC failure and shunt dependence.
\end{abstract}

METHODS A retrospective analysis was performed on a consecutive series of 48 infants with hydrocephalus who underwent ETV/CPC and also underwent preoperative echocardiography between 2007 and 2014. Comorbid cardiac abnormalities that are known to result in elevated right heart pressure were reviewed. Associations between ETVICPC success and the presence of pulmonary hypertension, right ventricular hypertrophy, left-to-right shunting, ventricular septal defect, or patent ductus arteriosus were determined using multivariate logistic regression analysis.

RESULTS Of the 48 children who met the inclusion criteria, ETVICPC failed in 31 (65\%). In univariate analysis, no single echocardiogram abnormality was associated with shunt failure, but the presence of 2 or more concurrent echocardiogram abnormalities was associated with ETV/CPC failure (17 [85\%] of 20 vs 14 [50\%] of 28, respectively; $p=0.018$ ). In multivariate logistic regression analysis, when the authors adjusted for the child's ETV success score, the presence of 2 abnormalities remained independently associated with poor outcome (2 or more echocardiogram abnormalities, OR $0.13,95 \% \mathrm{Cl} 0.01-0.7, \mathrm{p}=0.032$; ETV success score, OR 1.1, 95\% Cl 1-1.2, $\mathrm{p}=0.05$ ).

CONCLUSIONS In this study, cardiac abnormalities were inversely associated with the success of ETV/CPC in infants with hydrocephalus of prematurity. ETV/CPC might not be as efficacious in patients with significant cardiac anomalies. These results provide a basis for future efforts to stratify surgical candidacy for ETVICPC on the basis of comorbid abnormalities. Proper cardiac physiological pressure monitoring might help elucidate the relationship between cardiac abnormalities and hydrocephalus.

https://thejns.org/doi/abs/10.3171/2017.7.PEDS17132

KEY WORDS endoscopic third ventriculostomy; echocardiography; failure; hydrocephalus

$\mathrm{P}$ ROGRESSIVE hydrocephalus in infants often requires surgical intervention that frequently involves ventriculoperitoneal shunting. In an effort to reduce the need for a ventriculoperitoneal shunt (VPS) and potentially limit exposure to its associated complications in this population, endoscopic third ventriculostomy and choroid plexus cauterization (ETV/CPC) have been offered as a viable alternative to treat both obstructive and communicating hydrocephalus. ${ }^{9,10,21}$ Success rates of $40 \%-60 \%$ in controlling hydrocephalus 1 year after ETV/CPC in devel- oped countries were reported recently. ${ }^{15,19,21}$ Predictors of ETV outcome have traditionally been hypothesized and validated using the ETV success score (ETVSS), which uses cause, age, and shunting history to determine the likelihood of ETV success. ${ }^{8,12}$

Among neonatal comorbid conditions, congenital heart disease (CHD) might be implicated in the development and worsening of hydrocephalus in the 1st year of life. Some CHDs (e.g., pulmonary artery stenosis, tetralogy of Fallot, patent ductus arteriosus [PDA]) have been known to

ABBREVIATIONS CHD = congenital heart disease CPC = choroid plexus cauterization; $\mathrm{ETV}=$ endoscopic third ventriculostomy; ETVSS = ETV success score; PDA = patent ductus arteriosus; VPS = ventriculoperitoneal shunt.

SUBMITTED March 13, 2017. ACCEPTED July 19, 2017

INCLUDE WHEN CITING Published online November 10, 2017; DOI: 10.3171/2017.7.PEDS17132. 
increase central venous pressure and thereby impair CSF absorption gradients across the arachnoid granulations. Early suggestions of the relationship between the central venous system and hydrocephalus were noted more than 50 years ago in infants with right heart failure and vein of Galen malformation. In these infants, high intracranial venous pressure produced both hydrocephalus and secondary heart failure. ${ }^{2}$ This phenomenon was found even in infants with central venous occlusion/stenosis (superior vena cava), who then developed progressive communicating hydrocephalus. The increased venous pressure was thought to impair the CSF pulsatility and to impair CSF absorption gradients. ${ }^{6}$ In addition, infants with a single ventricle syndrome and higher documented central venous pressure have been noted to experience worsening intracranial pressure with increasing ventricular size. ${ }^{11}$ With these results in mind, the theory that congenital cardiac defects produce higher central venous pressure (and therefore worsen ventriculomegaly/hydrocephalus) should be investigated.

We hypothesized that the efficacy of ETV/CPC might be reduced in patients with concomitant CHD (arbitrarily defined in our study as 2 or more echocardiographic abnormalities), who might have elevated central venous pressure, and that the absorption of CSF therefore would be impaired. We retrospectively studied the relationship between CHD and ETV failure in consecutive hydrocephalic infants who were treated at our institutions with ETV/CPC for hydrocephalus of all causes. Because a large proportion of the hydrocephalic infants underwent screening echocardiography, we studied these patients' echocardiographic abnormalities and their association with ETV/ CPC outcome.

\section{Methods}

\section{Patient Selection}

After receiving institutional review board approval, we identified all ETV/CPC procedures performed on infants (less than 24 months old) at our 2 children's hospitals (Nicklaus Children's Hospital and Jackson Memorial Hospital) between January 2007 and December 2014. The patient cohort was sampled from a previously reported series of hydrocephalic infants who underwent ETV/CPC at our institutions. ${ }^{19}$ Patients with any cause of infantile hydrocephalus were included in this analysis. All patients in the series were treated primarily with ETV/CPC. The decision to use ETV/CPC was made at the discretion of the surgeon based on the anatomical feasibility, birth weight, and clinical status of each patient. The families were counseled on the known risks of ETV/CPC failure and the potential need for placement of a shunt afterward. ETV/CPC was performed with a rigid endoscope, as described previously. ${ }^{1,19}$

\section{Data Collection}

Patient demographic data, including gestational age, birth weight, head circumference, and cause of hydrocephalus, were collected. Relevant clinicoradiological data, including history of previous infections/meningitis, degree and morphology of hydrocephalus, and calculated
ETVSS, were collected also. ${ }^{8}$ The ETVSS (0-90) was calculated based on the description from the initial study by using age (score of $0-50)$, cause (0-30), and shunting history $(0-10)$.

Failure of ETV/CPC was defined as the primary outcome and determined based on the need for a VPS or other CSF-diversion procedure (repeat ETV) at any point within the follow-up period. Patients whose condition met progressive radiological (increasing ventriculomegaly) or clinical (increasing head circumference, bulging fontanelle/pseudomeningocele) criteria for symptomatic intracranial hypertension were considered for a repeat CSFdiversion procedure (primarily VPS), as determined by the treating neurosurgeon.

\section{Echocardiography}

Each patient included in our study underwent perioperative echocardiography as a component of clinical care. If echocardiography was performed multiple times, the echocardiogram temporally closest to the surgery date was selected for review. We evaluated relevant echocardiographic abnormalities that affect cardiac function, including left-to-right shift, atrial septal defect, ventricular septal defect, PDA, pulmonary hypertension, and right ventricular hypertrophy. Left-to-right shunts were noted only when seen on the echocardiogram by the attending pediatric cardiologist; these shunts were seen commonly in conjunction with a previous atrial/ventricular septal defect or PDA. Right ventricular pressure was estimated using a tricuspid regurgitant jet when identified with echocardiography based on the treating pediatric cardiologist's interpretation. Other anomalies, including total anomalous venous return and tetralogy of Fallot, were also documented when identified. Some patients required preoperative surgical correction (e.g., ligation of a persistent PDA, shunt placement) of their CHD before CSF diversion. In these cases, data from the most recent postintervention echocardiography were included.

\section{Statistical Analysis}

Descriptive statistics are presented as means with standard deviations and proportions for continuous and frequency data, respectively. Univariate analysis was performed using the Fisher exact test to determine differences in outcomes between children with and those without CHD. All variables with a $\mathrm{p}$ value less than 0.20 were entered into a multivariate logistic regression model to identify covariates independently associated with ETV/ $\mathrm{CPC}$ failure. All statistical analyses were performed using $\mathrm{R}$ software (R Foundation for Statistical Computing).

\section{Results}

Forty-eight children who underwent ETV/CPC and preoperative echocardiography were identified. Twenty children were included in the CHD group $(\geq 2$ abnormalities). We found no significant differences between baseline characteristics of the control infants and those of the infants with CHD (Table 1). The mean ages were 3.7 and 4.0 months for control infants and those with CHD, respectively $(p=0.71)$. The proportions of males in the 
TABLE 1. Baseline characteristics of the 48 infants who underwent preoperative echocardiography

\begin{tabular}{|c|c|c|c|}
\hline Characteristic & $\begin{array}{l}\text { Control Infants } \\
\qquad(n=28)\end{array}$ & $\begin{array}{c}\text { Infants w/ CHD } \\
(n=20)\end{array}$ & $\begin{array}{c}\mathrm{p} \\
\text { Value }\end{array}$ \\
\hline Age in mos (IQR) & $3.7(1.4-4.8)$ & $4.0(2.2-5.1)$ & $0.71^{*}$ \\
\hline Sex (no. [\%]) & & & $0.77^{*}$ \\
\hline Male & $16(57)$ & $10(50)$ & \\
\hline Female & $12(43)$ & $10(50)$ & \\
\hline ETVSS (median [range]) & $40(10-70)$ & $40(30-70)$ & $0.95 \dagger$ \\
\hline $\begin{array}{l}\text { Prepontine space in mm } \\
\text { (mean [IQR]) }\end{array}$ & $3.4(1.7-4.9)$ & $2.7(1.1-3.2)$ & $0.36 \dagger$ \\
\hline Etiology (no. [\%]) & & & $0.29 \ddagger$ \\
\hline IVH prematurity & $16(57)$ & $16(80)$ & \\
\hline Aqueductal stenosis & $5(18)$ & $3(15)$ & \\
\hline Myelomeningocele & $0(0)$ & $1(5)$ & \\
\hline Dandy Walker Type IV & $1(3.6)$ & $0(0)$ & \\
\hline $\begin{array}{l}\text { Congenital communicat- } \\
\text { ing hydrocephalus }\end{array}$ & $2(7.1)$ & $0(0)$ & \\
\hline Previous infection & $2(7.1)$ & $0(0)$ & \\
\hline Other & $2(7.1)$ & $0(0)$ & \\
\hline Complications (no. [\%]) & $2(7.1)$ & $2(10)$ & - \\
\hline Degree of CPC (\% bilateral) & $25(89)$ & $18(90)$ & $1.0^{*}$ \\
\hline $\begin{array}{l}\text { Follow-up duration in mos } \\
\text { (mean [range]) }\end{array}$ & $22.6(0.3-60)$ & $30.4(0.5-72)$ & $0.2 \dagger$ \\
\hline
\end{tabular}

control (57\%) and CHD (50\%) groups were also similar $(\mathrm{p}=0.77)$. The median ETVSS in both groups was $40(\mathrm{p}$ $=0.95$ ). Intraventricular hemorrhage of prematurity was the most common cause of hydrocephalus in both groups (57\% [control group] and 80\% [CHD group]). Mean measurements of the prepontine space (distance between the dorsum sellae and basilar artery) from preoperative MR images were also similar in the control and CHD groups (3.4 and $2.7 \mathrm{~mm}$, respectively) $(\mathrm{p}=0.36)$.

The most common echocardiographic abnormality in the infants with CHD was a left-to-right shunt (90\%) and presence of a PDA (85\%) (Table 2). Four (20\%) of the 20 infants with CHD had evidence of right ventricular hypertrophy $(\mathrm{p}=0.025)$, compared with none of the infants in the control group. Among the 48 infants, 18 (11 controls, 7 with CHD) had evidence of tricuspid regurgitant jet on a preoperative echocardiogram, which was associated with a nonsignificantly higher proportion of patients with elevated right ventricular pressure $(>20 \mathrm{~mm} \mathrm{Hg})$ in the CHD group $(57.1 \%$ [CHD group] vs $18.2 \%$ [control group]; $\mathrm{p}=$ $0.14)$

Of the 48 children, 31 (65\%) experienced ETV/CPC failure. The difference in the occurrence of failure between the 2 groups was significant (17 of [85\%] 20 in the CHD group vs 14 [50\%] of 28 in the control group; $\mathrm{p}=$ 0.016 ) (Table 3). In univariate analysis, no single echocardiographic abnormality was associated with shunt failure,
TABLE 2. Echocardiographic abnormalities in control infants and in infants with CHD

\begin{tabular}{lccc}
\hline \multicolumn{1}{c}{ Abnormality } & $\begin{array}{c}\text { Control Infants } \\
(\mathrm{n}=28)(\mathrm{no} .[\%])\end{array}$ & $\begin{array}{c}\text { Infants w/ CHD } \\
(\mathrm{n}=20)(\mathrm{no} .[\%])\end{array}$ & $\begin{array}{c}\mathrm{p} \\
\text { Value }\end{array}$ \\
\hline Left-to-right shunt & $3(10.7)$ & $18(90)$ & $<0.0001$ \\
\hline PDA & $0(0)$ & $17(85)$ & $<0.0001$ \\
\hline ASD/VSD & $0(0)$ & $6(30)$ & 0.016 \\
\hline RVH & $0(0)$ & $4(20)$ & 0.025 \\
\hline Tricuspid gradient $>20$ & $2 / 11(18.2)$ & $4 / 7(57.1)$ & 0.14 \\
\hline
\end{tabular}

$\mathrm{ASD} / \mathrm{VSD}=$ atrial septal defect/ventricular septal defect; $\mathrm{RVH}=$ right ventricular hypertrophy.

although infant age $(\mathrm{p}<0.001)$ and ETVSS $(\mathrm{p}=0.016)$ were significantly associated with the success of ETV/ CPC in univariate analysis (Table 4). We found no significant association between the prepontine space and ETV success $(p=0.13)$. The presence of 2 or more concurrent echocardiogram abnormalities was associated with ETV/ CPC failure $(\mathrm{p}=0.018)$.

Covariates with a $\mathrm{p}$ value less than 0.20 in the univariate analysis were entered into a multivariate model. Because of multicollinearity between age and ETVSS, which incorporates age in its derivation, only the ETVSS was included in the logistic regression analysis. In multivariate logistic regression analysis, in which we adjusted for the children's ETVSS, the presence of 2 abnormalities remained independently associated with outcome (2 or more echocardiogram abnormalities, OR $0.13,95 \%$ CI $0.01-0.7, \mathrm{p}=0.032$; ETVSS, OR 1.1, 95\% CI 1-1.2, $\mathrm{p}=$ $0.05)$ (Table 5).

\section{Discussion}

In the current exploratory analysis, we found that having multiple echocardiographic abnormalities was associated with ETV failure in infants with hydrocephalus of prematurity who underwent ETV/CPC. These findings support our hypothesis that elevated venous pressure, caused by comorbid cardiac disease, might predispose the patient to ETV/CPC failure and shunt dependence.

\section{Infantile CSF Absorption}

The pathophysiology of infantile hydrocephalus remains challenging, because many children present with a combination of obstructive and malabsorptive mechanisms contributing to their hydrocephalus. In these patients, ETV/CPC has been proposed to serve as a surrogate to avoid the need for shunt placement in infants;

TABLE 3. Differences in outcomes between control infants and infants with CHD, calculated by the Fisher exact test

\begin{tabular}{lccc}
\hline Outcome & $\begin{array}{c}\text { Control Infants } \\
(n=28)(n o .[\%])\end{array}$ & $\begin{array}{c}\text { Infants w/ CHD } \\
(n=20)(n o . ~[\%])\end{array}$ & $\begin{array}{c}p \\
\text { Value }\end{array}$ \\
\hline ETV failure & $14(50)$ & $17(85)$ & 0.016 \\
\hline Death & 1 & 1 & 1.0 \\
\hline
\end{tabular}


TABLE 4. Univariate (logistic regression) analysis of risk factors for ETV success

\begin{tabular}{lcrl}
\hline \multicolumn{1}{c}{ Risk Factor } & OR & $95 \% \mathrm{Cl}$ & $\mathrm{p} \mathrm{Value}$ \\
\hline Age & 1.4 & $1.1-1.8$ & 0.001 \\
\hline Sex & 0.9 & $0.3-3.1$ & 0.9 \\
\hline ETVSS & 1.1 & $1.0-1.2$ & 0.016 \\
\hline Prepontine space & 1.2 & $0.9-1.7$ & 0.13 \\
\hline CHD ( $\geq 2$ abnormalities) & 0.2 & $0.04-0.7$ & 0.018 \\
\hline Extent of CPC & 0.8 & $0.1-6.6$ & 0.82 \\
\hline IVH of prematurity & 0.9 & $0.3-3.1$ & 0.83 \\
\hline
\end{tabular}

it offers an approximately $50 \%$ success rate. ${ }^{19}$ The ETV/ CPC procedure attempts to bypass the CSF pathway obstruction (ETV) and reduce production (CPC), but its success can be predicated on the premise that CSF absorption pathways function appropriately. The physiology of CSF dynamics was described as early as the 1850s when Faivre initially proposed the role of the choroid plexus in CSF production. ${ }^{17}$ Over the next several decades, Cushing ${ }^{3}$ and Dandy ${ }^{4}$ expanded the understanding of CSF absorption by suggesting that CSF is absorbed across the arachnoid villi. In the 1930 s, Weed ${ }^{18}$ expanded this theory by suggesting that this CSF absorption depends on hydrostatic pressure gradients between the CSF compartment, the arachnoid granulations, and the dural venous sinuses. These results have developed into our current, yet limited, understanding of CSF production and absorption.

Impaired CSF absorption across the arachnoid villi can be a result of a diminished gradient between CSF pressure and sinovenous outflow pressure. If the hydrostatic gradient described by Weed and Dandy is disrupted, CSF might not be absorbed adequately. This phenomenon has been described in patients with pseudotumor cerebri in whom cardiac disease results in higher central venous pressure (and therefore higher dural sinus pressure) and venous outflow obstruction as a result of major venous thrombosis with worsening intracranial hypertension. . $^{516}$ A similar mechanism has been noted in infants with congenital single ventricle who develop ventriculomegaly and hydrocephalus after cardiac shunting procedures. These infants were noted to have increasing ventricular size with concomitant increases in central venous pressure after cardiac intervention, which suggests the importance of the CSF absorption gradient and CSF pulsatility in infants. ${ }^{11}$ As a result, we hypothesized that hydrocephalic infants with cardiac disease (a proxy for increased central venous pressure) were more prone to ETV/CPC failure.

\section{CHD and Its Implications}

In our small study of 48 children, CHD (arbitrarily defined as 2 or more echocardiographic abnormalities) was associated with a significant risk of ETV/CPC failure, independent of ETVSS. The cardiac conditions assessed were surrogates for increased right-sided heart pressure and therefore potentially higher central venous pressure. Atrial septal defects, ventricular septal defects, pulmonary hypertension, PDA, and right ventricular hypertrophy all
TABLE 5. Multivariate (logistic regression) analysis of risk factors for ETV success

\begin{tabular}{llrl}
\hline \multicolumn{1}{c}{ Risk Factor } & OR & $95 \% \mathrm{Cl}$ & \multicolumn{1}{c}{$\mathrm{p} \mathrm{Value}^{*}$} \\
\hline ETVSS & 1.1 & $1-1.2$ & 0.05 \\
\hline Prepontine space & 1.1 & $0.8-1.6$ & 0.47 \\
\hline CHD $(\geq 2$ abnormalities $)$ & 0.13 & $0.01-0.7$ & 0.032 \\
\hline
\end{tabular}

* Threshold for inclusion was $p<0.1$ in univariate analysis.

have been found to affect right heart pressure adversely.,13 One of the main assumptions of our study was that these cardiac abnormalities would alter cardiovascular dynamics to potentially elevate right heart pressure and eventually central venous pressure. The schematic in Fig. 1 shows the theoretical relationship between CHD and hydrocephalus. Right heart pressure cannot be assessed directly through an echocardiogram and can only be estimated in some patients with a tricuspid regurgitant jet. The absence of a regurgitant jet does not necessarily equate to a lack of right ventricular hypertension. Right ventricular pressure can be estimated by the sum of the right atrial pressure and the tricuspid gradient; right ventricular pressure greater than half of the systemic blood pressure is indicative of right-sided hypertension. In our study, we were unable to measure right ventricular pressure directly; however, the tricuspid regurgitant jet was higher in a greater percentage of infants with CHD than in the controls, which suggests a relationship between echocardiographic abnormalities and elevated right heart pressure.

\section{Etiology}

The most common cause of hydrocephalus in our cohort was posthemorrhagic hydrocephalus of prematurity, which is consistent with other series of patients who underwent ETV/CPC in a developed nation. In these infants, the ETV/CPC success rate was approximately $35 \%$, which is lower than the 50\% shunt-freedom rate reported in the literature. We hypothesize that our study might have been subject to an inherent selection bias given the study criteria. Patients with predominantly obstructive hydrocephalus typically respond better to ETV/CPC than patients with communicating hydrocephalus. ${ }^{15,20}$ Moreover, the ETVSS uses etiology to predict ETV/CPC success (aqueductal stenosis scores higher than hemorrhagic hydrocephalus of prematurity). The ETVSS was also found in our study to be an independent predictor of ETV/CPC success, which helps validate our expected results. In our study, we found no significant difference in hydrocephalus etiologies between the CHD and control groups.

\section{Surgical Repair}

In some $(n=9)$ infants with severe CHD, surgical repair of the cardiac defect was necessary. In these patients, we observed an improvement in echocardiographic abnormalities on serial examinations, and some $(n=2)$ patients experienced resolution of elevated right heart pressure. Nevertheless, many of the patients had residual echocardiographic abnormalities and ETV/CPC failed, which 


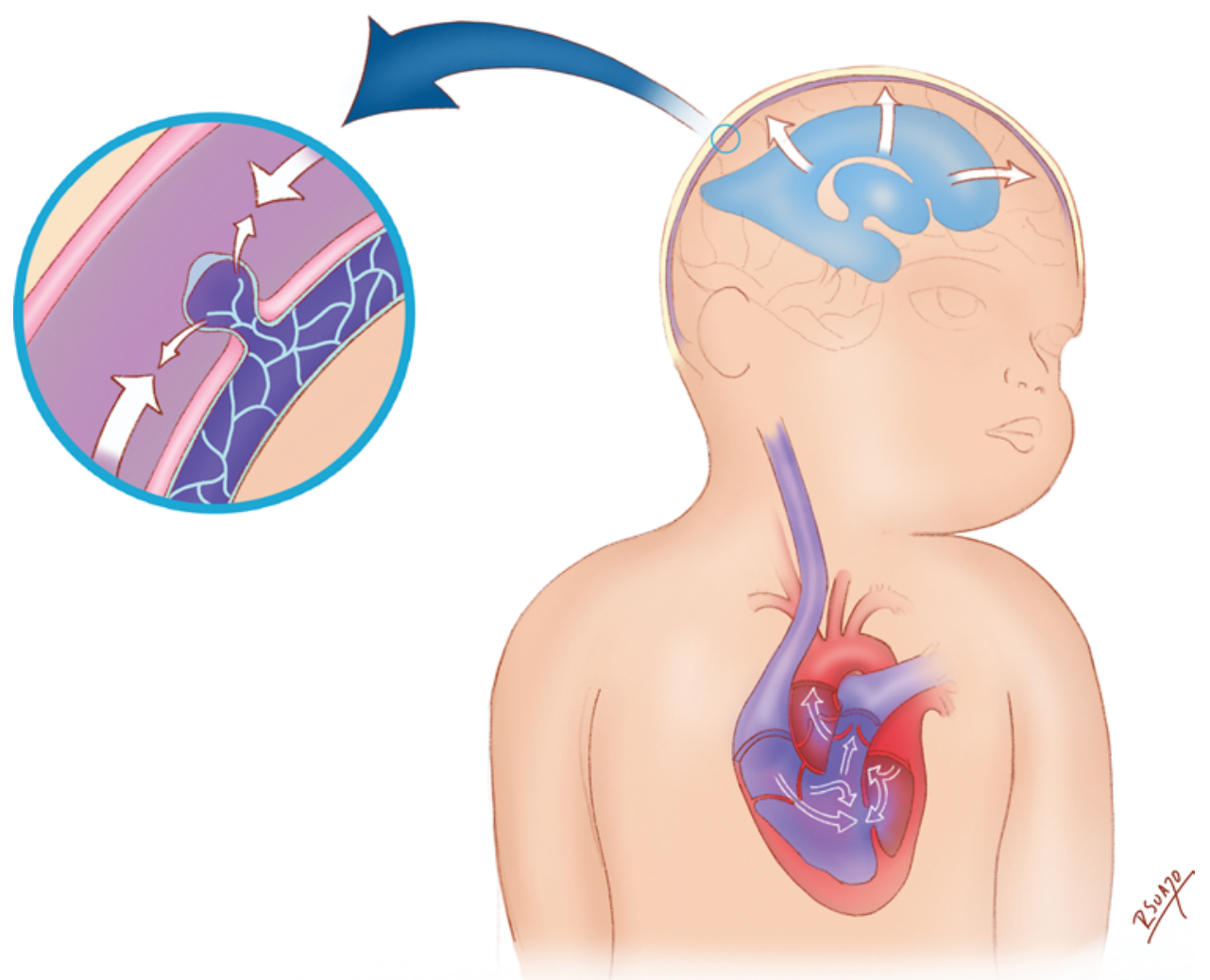

FIG. 1. Artist's illustration showing the theoretical relationship between CHD and hydrocephalus. Copyright Toba N. Niazi. Published with permission.

suggests that the continued presence of cardiac anomalies despite surgical correction of other abnormalities might be associated with ETV/CPC failure. These anatomical defects that persist after surgery might indicate an $a b-$ normal cardiac physiology with increased central venous pressure. In our study, we reviewed echocardiograms that were temporally closest to the day of surgery to avoid misrepresentation of the infant's preoperative cardiac status.

\section{Limitations and Future Directions}

Compared with larger ETV/CPC series, our study was limited by the relatively small sample size $(n=48)$. Despite this limitation, we discerned a statistical and clearly clinical difference in outcomes between infants with CHD and control infants. A larger prospective series of infants who are screened for cardiac disease before ETV/CPC could further elucidate the relationship between CHD and infantile hydrocephalus. Because the inclusion criteria for our study required the presence of a preoperative echocardiogram, an inherent selection bias remains in our cohort in that patients with suspected cardiac disease were preferentially included. Therefore, our definition of CHD might also be strengthened with more rigorous criteria (e.g., need for surgical intervention, presence of cardiac syndromes [tetralogy of Fallot], documented elevated central venous pressure, etc.). In addition, because echocardiograms can be used only indirectly to estimate central venous pressure, more invasive direct measurements of venous pres- sure (e.g., invasive venous monitoring, transesophageal echocardiography) would be necessary to confirm our hypothesis but also would subject infants to potentially harmful procedures. Use of the tricuspid regurgitant jet is also limited in its ability to estimate the severity of right ventricular pressure, because many infants with pulmonary arterial hypertension do not have tricuspid regurgitation. Therefore, a more robust and more prevalent surrogate marker of elevated central venous pressure might be needed. In addition, it is important to acknowledge that ventriculomegaly associated with elevated central venous pressure can manifest as a benign course that might be misinterpreted as ETV success; however, in our series, ETVs were performed only on patients with symptomatic hydrocephalus (increasing head circumference, symptomatology, or radiographic progression). Therefore, we hoped to exclude infants with ventriculomegaly without hydrocephalus. Last, our study was limited by the nature of many retrospective studies, including 1) the lack of long-term follow-up in certain infants, 2) selection bias for ETV/CPC (infants with severe CHD who were receiving blood pressure/ventilator support were not taken to the operating room until they were stabilized), and 3) informational bias that might be inherent when interpreting echocardiograms of infants (e.g., estimating right ventricular pressure, multiple cardiologists). Despite these biases, the ETV/CPC failure rate (50\%) in our control group was similar to that reported in other North American series 
reports (43-62\%). ${ }^{9} 15,19$ Our results encourage the design of a prospective study for assessing the cardiac status of hydrocephalic infants before ETV/CPC, which could include additional noninvasive monitoring of pulmonary artery pressure (e.g., electric impedance tomography, advanced Doppler echocardiography), which might estimate right ventricular pressure reliably. ${ }^{14}$

\section{Conclusions}

We have shown here an association between echocardiographic abnormalities and ETV/CPC failure. Our findings indicate an association between CHD and ventriculomegaly. We suggest that these congenital heart abnormalities can alter normal cardiac physiology, potentially increasing central venous pressure. Although neonates are increasingly treated with ETV/CPC for hydrocephalus related to prematurity, our study results suggest a role for patient stratification on the basis of multiple echocardiographic abnormalities. Future prospective studies with a more directed approach to central venous pressure monitoring might be helpful in elucidating the relationship between persistent hydrocephalus, elevated right heart pressure, and cardiac defects.

\section{References}

1. Chamiraju P, Bhatia S, Sandberg DI, Ragheb J: Endoscopic third ventriculostomy and choroid plexus cauterization in posthemorrhagic hydrocephalus of prematurity. J Neurosurg Pediatr 13:433-439, 2014

2. Cronqvist S, Granholm L, Lundström NR: Hydrocephalus and congestive heart failure caused by intracranial arteriovenous malformations in infants. J Neurosurg 36:249-254, 1972

3. Cushing H: Studies on the cerebro-spinal fluid. I. Introduction. J Med Res 31:1-19, 1914

4. Dandy WE: Where is cerebrospinal fluid absorbed? JAMA 92:2012-2014, 1929

5. Fuchs H, Singh D, Greene C Jr, Ross-Ascuitto N, Ascuitto R: Pseudotumor cerebri associated with modified Fontan anatomy. Pediatr Cardiol 34:1932-1934, 2013

6. Hooper R: Hydrocephalus and obstruction of the superior vena cava in infancy. Clinical study of the relationship between cerebrospinal fluid pressure and venous pressure. Pediatrics 28:792-799, 1961

7. Koestenberger M, Burmas A, Ravekes W, Avian A, Gamillscheg A, Grangl G, et al: Echocardiographic reference values for right atrial size in children with and without atrial septal defects or pulmonary hypertension. Pediatr Cardiol 37:686-695, 2016

8. Kulkarni AV, Drake JM, Kestle JR, Mallucci CL, Sgouros $\mathrm{S}$, Constantini S: Predicting who will benefit from endoscopic third ventriculostomy compared with shunt insertion in childhood hydrocephalus using the ETV success score. J Neurosurg Pediatr 6:310-315, 2010

9. Kulkarni AV, Riva-Cambrin J, Browd SR, Drake JM, Holubkov R, Kestle JR, et al: Endoscopic third ventriculostomy and choroid plexus cauterization in infants with hydrocephalus: a retrospective Hydrocephalus Clinical Research Network study. J Neurosurg Pediatr 14:224-229, 2014

10. Kulkarni AV, Warf BC, Drake JM, Mallucci CL, Sgouros S, Constantini S: Surgery for hydrocephalus in sub-Saharan Africa versus developed nations: a risk-adjusted comparison of outcome. Childs Nerv Syst 26:1711-1717, 2010
11. Morgan CD, Wolf MS, Le TM, Shannon CN, Wellons JC III, Mettler BA: Cerebral ventriculomegaly after the bidirectional Glenn (BDG) shunt: a single-institution retrospective analysis. Childs Nerv Syst 31:2131-2134, 2015

12. Naftel RP, Reed GT, Kulkarni AV, Wellons JC: Evaluating the Children's Hospital of Alabama endoscopic third ventriculostomy experience using the Endoscopic Third Ventriculostomy Success Score: an external validation study. J Neurosurg Pediatr 8:494-501, 2011

13. Patel SG, Woolman P, Li L, Craft M, Danford DA, Kutty S: Relation of right atrial volume, systemic venous dimensions, and flow patterns to right atrial pressure in infants and children. Am J Cardiol 119:1473-1478, 2017

14. Proença M, Braun F, Solà J, Adler A, Lemay M, Thiran JP, et al: Non-invasive monitoring of pulmonary artery pressure from timing information by EIT: experimental evaluation during induced hypoxia. Physiol Meas 37:713-726, 2016

15. Stone SS, Warf BC: Combined endoscopic third ventriculostomy and choroid plexus cauterization as primary treatment for infant hydrocephalus: a prospective North American series. J Neurosurg Pediatr 14:439-446, 2014

16. Sugerman HJ, DeMaria EJ, Felton WL III, Nakatsuka M, Sismanis A: Increased intra-abdominal pressure and cardiac filling pressures in obesity-associated pseudotumor cerebri. Neurology 49:507-511, 1997

17. Symss NP, Oi S: Theories of cerebrospinal fluid dynamics and hydrocephalus: historical trend. J Neurosurg Pediatr 11:170-177, 2013

18. Weed LH: Meninges and cerebrospinal fluid. J Anat 72:181215, 1938

19. Weil AG, Fallah A, Chamiraju P, Ragheb J, Bhatia S: Endoscopic third ventriculostomy and choroid plexus cauterization with a rigid neuroendoscope in infants with hydrocephalus. J Neurosurg Pediatr 17:163-173, 2016

20. Weil AG, Westwick H, Wang S, Alotaibi NM, Elkaim L, Ibrahim GM, et al: Efficacy and safety of endoscopic third ventriculostomy and choroid plexus cauterization for infantile hydrocephalus: a systematic review and meta-analysis. Childs Nerv Syst 32:2119-2131, 2016

21. Zandian A, Haffner M, Johnson J, Rozzelle CJ, Tubbs RS, Loukas M: Endoscopic third ventriculostomy with/without choroid plexus cauterization for hydrocephalus due to hemorrhage, infection, Dandy-Walker malformation, and neural tube defect: a meta-analysis. Childs Nerv Syst 30:571-578, 2014

\section{Disclosures}

The authors report no conflict of interest concerning the materials or methods used in this study or the findings specified in this paper.

\section{Author Contributions}

Conception and design: Shah, Bhatia. Acquisition of data: Shah, Ibrahim. Analysis and interpretation of data: Shah, Ibrahim, Sasaki, Bhatia. Drafting the article: Shah, Ibrahim, Sasaki. Critically revising the article: all authors. Reviewed submitted version of manuscript: Niazi, Shah, Ibrahim, Ragheb, Bhatia. Statistical analysis: Shah, Ibrahim. Administrative/technical/material support: Niazi, Shah, Ibrahim, Ragheb. Study supervision: Niazi, Ibrahim, Sasaki, Ragheb, Bhatia.

\section{Correspondence}

Toba N. Niazi, Department of Neurological Surgery, Nicklaus Children's Hospital, 3100 SW 62nd Ave., Miami, FL 33136. email: toba.niazi@mch.com. 